\title{
Hematologic Emergencies
}

\author{
Gianfilippo Nifosì \\ Hemato-Oncologic Clinic, Department of Internal Medicine, University Hospital Center Brugmann, Bruxelles, \\ Belgium \\ Email: gianfilippo.nifosi@chu-brugmann.be
}

Received 29 June 2016; accepted 9 September 2016; published 12 September 2016

Copyright (C) 2016 by author and Scientific Research Publishing Inc.

This work is licensed under the Creative Commons Attribution International License (CC BY).

http://creativecommons.org/licenses/by/4.0/

(c) (i) Open Access

\section{Abstract}

Hematological emergencies are defined as acute life-threatening conditions in hematological diseases due to benign blood disorders or hematological malignancies or as a result of its treatment. This article deals of the most common emergencies encountered by hematologists. Emerging clinical situations, predictable or not, are common in the treatment of hematological diseases. However, the rapid development of new targeted drugs in cancer medicine has led to an increase in chronically ill cancer and hematology patients, who are being treated aggressively despite significant comorbidities and higher age. This development will lead to an increase in the number of hematologic emergencies, and these patients will be seen by various specialists. The knowledge of most hematological emergencies is crucial in daily practice. In this review, we discuss the epidemiology, pathophysiology and clinical with an emphasis on the early diagnosis and treatment of these conditions that can significantly affect the patient's clinical outcome.

\section{Keywords}

Urgency, Hematological Diseases, Clinical Practice

\section{Introduction}

In Hematology, we can have various emergencies, either directly related to hematologic disease or complications of them or their treatment. In Table 1, we distinguish emergencies in the course of benign and malignant hematological diseases.

\section{Autoimmune Haemolytic Anemia Induced by Drugs}

Autoimmune haemolytic anemia induced by drugs (DHIA) is a form of autoimmune haemolytic anemia in response to drug administration, through various mechanisms, including the producion of autoantibodies and absorption of immune complexes and complement on the cell membrane [1]. This is a rare disease whose annual 
Table 1. Main medical emergencies in hematology.

\begin{tabular}{cc}
\hline Benign hematologic diseases & Hematological malignancies \\
\hline Autoimmune haemolytic anemia induced by drugs & Hypercalcemia \\
Thrombotic thrombocytopenic purpura & Hyperleukocytosis \\
Sickle cell crisis & Hyperviscosity \\
& Acute tumor lysis syndrome \\
& Spinal cord compression \\
\hline
\end{tabular}

incidence is estimated at 1/1,000,000. It's more common in adults and rarely in children. This is important because it has a $10 \%-20 \%$ mortality and that can get to $50 \%$ among young people. The most frequently implicated drugs are antibiotics: cephalosporins (cefotetan and ceftriaxone) and piperacillin. Fludarabine is involved if used in monotherapy. Finally, do not forget hydrocortisone. Clinically is characterized by severe acute anemia without alterations of leukocytes and platelets, reticulocytes, $\mathrm{LDH}$ and indirect bilirubin increased. The direct Coombs test is positive (IgG or IgM and C'). Stopping the causative drug can regress or disappear symptoms. The intravenous administration of immunoglobulins and corticosteroides drugs ( $1-2 \mathrm{mg} / \mathrm{kg} / \mathrm{day}$ of methylprednisolone) can also be considered to reduce the immune response. A blood transfusion may be necessary in severe cases that endanger life.

\section{Thrombotic Thrombocytopenic Purpura (Moschowitz Disease)}

Thrombotic thrombocytopenic purpura (TTP) is a severe occlusive disease of the small vessels (microangiopathy) characterized by systemic platelet aggregation, ischemic damage, marked thrombocytopenia with megakaryocytes increased in the bone marrow and fragmentation of red blood cells. Classically TTP is characterized by five signs/symptoms: thrombocytopenia; microangiopathic haemolytic anemia; neurological abnormalities; kidney failure; and fever.

More recently there have been attempts to simplify the diagnostic criteria in three major signs/symptoms whose simultaneous presence should suggest the diagnosis [2] [3]:

1) thrombocytopenia;

2) presence of schistocytes ( $>1 \%$ of the total red blood cells);

3 ) values of lactate dehydrogenase (LDH) extremely high.

Two forms of Moschowitz disease are known as congenital and acquired. The latter is definitely more frequent.

At the origin of congenital TTP there is an alteration in the ADAMTS 13 gene that causes reduced or absent ADAMTS 13 enzyme synthesis. Conversely, in the acquired TTP, ADAMTS 13 enzyme is inhibited or reduced by the presence of autoantibodies. The conditions associated with acquired TTP are: pregnancy, infections, systemic lupus erythematous, drugs, organ and bone marrow transplant, and malignancy.

A special case is that observed in the course of infection by Escherichia coli producer of shigatossina. The clinical picture is characterized by diarrhea associated with haemolytic uremic syndrome.

Several drugs can be involved: ticlopidine, clopidogrel, fluorochinolones, cyclosporine [4], tacrolimus and chemotherapy drugs [5].

In related forms to infection or drugs, rarely ADAMTS 13 is reduced.

ADAMTS 13 is a metalloproteinase that degrades von Willebrand factor (vWF). The excess of multimers unusually long of von Willebrand factor (ULVWF) promote platelet aggregation and platelet-dependent microvascular thrombosis leading partial or complete occlusion of the microcirculation with the resulting in symptoms/signs:

1) the red cells, crossing through the turbulence induced by platelets aggregated, are fragmented and damaged giving rise to schistocytes; they are highlighted using peripheral blood smears and are considered positive when they are $>1 \%$ of the total red blood cells;

2) in turn, platelet aggregation in the microcirculation resulting in a fall in platelet counts;

3 ) the partial or complete occlusion of the microcirculation causes then the symptoms associated with the or- 
gan suffering.

A dosage of ADAMTS 13 less than 5\% is diagnostic. This allows you to make the differential diagnosis with other diseases, which you should distinguished from the TTP, but that also exhibit a decline, though less, of ADAMTS 13 such as, for example, the disseminated intravascular coagulation and chronic liver disease.

ADAMTS 13 has prognostic value: TTP with low levels of ADAMTS 13 has more probability to respond to treatment than a TTP with normal values of ADAMTS 13.

The standard treatment of TTP relies upon therapeutic plasma exchange (PLEX) to 40 - $60 \mathrm{ml} / \mathrm{Kg} / \mathrm{day}$, which replaces ADAMTS 13 and removes circulating inhibitory autoantibodies. Mortality from TTP was previously very high $(>90 \%)$, but has decreased to $<20 \%$ with PLEX treatment $[6]$.

If the plasma exchange is not available, or still waiting to subject the patient to such a procedure, it is recommended plasma infusion up to $30 \mathrm{~mL} / \mathrm{kg} /$ day associating methylprednisolone to the dose of 1 to $2 \mathrm{mg} / \mathrm{kg}$.

$10 \%-42 \%$ of patients not respond to PLEX and corticosteroids and require further therapy.

Treatment options for TTP refractory included: corticosteroids, twice-daily PEX, cyclosporine, cyclophosphamide, vincristine and splenectomy.

Case reports and small studies have documented success with the use of rituximab in the treatment of refractory cases, with substantial improvement in the outcome. The majority of patients respond to a single dose of rituximab and improvement is seen within 2 weeks, but the exact dosage and duration of rituximab therapy is unknown [7].

In the last few years, new potential therapies for refractory TTP have shown promise, including bortezomib and $\mathrm{N}$-acetylcysteine (NAC) [8].

Recently, caplacizumab, an anti-von Willebrand factor humanized single-variable-domain immunoglobulin (Nanobody), that inhibits the interaction between ULVWF and platelets, in a phase 2 study, it has been shown a faster resolution of the acute TTP episode than placebo, though associated with an increased tendency toward bleeding [9].

It was recently synthesized a protease recombinant ADAMTS 13 which certainly will represent an important treatment strategy in the future.

\section{Sickle Cell Crisis}

The interest for this condition originates from growing number of patients with sickle cell disease (SCD) that come to our observation, secondary to migration waves. The fundamental primary lesion of sickle cell disease is the polymerization of deoxygenated $\mathrm{HbS}$ resulting in the deformation of red blood cells (RBCs) into pathognomonic sickle-shaped cells. RBC sickling causes structural membrane damage leading to premature clearing of the affected cells by the reticuloendothelial system, abnormal expression of surface adhesion molecules and impaired blood rheology. Haemolysis and alterations of blood rheology stemming from a high proportion of circulating sickle cells lead to a complex inflammatory cascade affecting all components of Virchow's triad and resulting in endothelial dysfunction, hyperviscosity, and hemostatic activation. Whereas RBC sickling is incessant and necessary for vaso-occlusion, vaso-occlusive crises (VOCs) are episodics, caused by events triggers, such as hypoxia, metabolic acidosis, intense exercise and infections [10].

Acute painful crises are the expression of the vaso-occlusive episodes charged to the bone microcirculation with ischemic bone marrow suffering. This picture may then be complicated with bone fractures and/or fat embolism. Patients with sickle cell disease are frequently visited the emergency department as a result of acute painful crises. Early and aggressive management is a priority: effective pain control, adequate fluid infusion, adequate oxygen therapy, correction of metabolic acidosis and transfusion of red blood cells.

Acute chest syndrome (ACS) is a lung injury syndrome that can complicate hospitalizations for acute VOC or appear de novo as a presenting admission diagnosis. It is also associated with prolonged hospitalization (on average, 10.5 days) decreased long-term survival and increased risk of developing chronic lung disease. The clinical presentation includes fever, chest pain, dyspnea and decreased oxygen saturation. Radiologically, it highlights multilobar involvement or complete lung segment consolidation. Several causes of ACS have been identified, although in most cases the direct insult is unknown. Infections, fat embolization, in situ pulmonary thrombosis and hypoventilation have been hypothesized. RBC transfusions aimed at increasing oxygen delivery and reducing the percentage of $\mathrm{HbS}$ should be administered as simple or automated exchange transfusions (erythrocytapheresis). Furthermore, although volume expansion with crystalloids is important in SCD, it should be deli- 
vered at a cautious rate in these hemodynamically vulnerable patients, while RBC transfusions may need to be followed by diuretic therapy. Deep vein thrombosis prophylaxis should be instituted routinely in patients with ACS, and clinical research is ongoing to establish whether systemic anticoagulation can have a beneficial effect both in preventing thrombosis and improving the ACS disease course [11].

\section{Disseminated Intravascular Coagulation}

Disseminated intravascular coagulation (DIC) is an acquired disease brought about by activation of blood coagulation without apparent reason, expression of an underlying illness that can cause severe organ damage.

The causes of DIC can be classified into several groups of disorders: infections, obstetrics, toxics, immunologicals, inflammatory, vascular, malignancy and liver diseases.

In the field of hematology, it may be the event of onset or evolution of acute leukemias, particularly acute promyelocytic leukemia (APL), acute lymphoblastic leukemia and occasionally acute myeloblastic leukemia.

Whatever the primary cause, the coagulation cascade is activated in such a way that the prothrombotic products which they derived far exceed the capacity of clearance of reticuloendothelial system and the capacity of the fibrinolytic system. In this situation these products, free from circulating thrombin inhibitors, may exert an unbalanced effect on the fibrinogen reducing it to fibrin, platelet activating and factors V, VIII and XIII and then completing the coagulation process with production of thrombi in the blood stream, mainly in the microvasculature. The hyperbolic consumption uncontrolled of clotting factors in the formation of thrombus door, the other side, to an absolute deficiency of these factors and, therefore, cause of hemorrhagic manifestations, even dramatic, next to thrombotic processes [12].

Finally, thrombin, along with thrombomodulin, active protein $\mathrm{C}$ which in turn activates the fibrinolytic system and inhibits the factors V and VIII activated. The fibrinolytic system is also activated by the endothelial release of tPA which in turn active plasmin. Plasmin, not blocked by his physiological inhibitors, is free to degrade the factors V, VIII, XIII, the fibrin and fibrinogen same, so as to produce in large excess fragments of fibrinogen degradation.

The diagnosis of DIC requires an appropriate clinical picture and some alterations in coagulation tests. An diagnostic algorithm for DIC has been proposed by International Society of Thrombosis and Haemostasis (ISTH) [13] [14]:

1) Risk assessment: does the patient have an underlying disorder known to be associated with overt DIC?

- If yes: processed

- If no: do not use this algorithm

2) Order global coagulation tests: prothrombine time, platelet count, fibrinogen, fibrin related marker.

3) Score the test results:

- Platelet count: $>100 \times 10^{9} / l=0,<100 \times 10^{9} / l=1,<50 \times 10^{9} / l=2$.

- Elevated fibrin marker (e.g., D-dimer, fibrin related marker): no increase $=0$, moderate increase $=2$, strong increase $=3$.

- Prolonged prothrombine time: $<3 s=0,>3$ but $<6 s=1,>6 s=2$.

- Fibrinogen level: $>1 \mathrm{~g} / \mathrm{l}=0,<1 \mathrm{~g} / \mathrm{l}=1$.

4) Add the score together.

5) Analyze the final score:

- $\geq 5$ compatible of overt DIC: repeat score daily.

- <5 suggestive for no-overt DIC: repeat next $1-2 d$.

The diagnosis of DIC is made by exclusion of other conditions such as: Hemodilution by bleeding/massive transfusion; TTP; Heparin-induced thrombocytopenia; Vitamin K deficiency; and Liver failure. Pathogenetic events responsible of DIC can be arrested by an appropriate treatment of the underlying condition, even if the supportive treatment represents a key moment in the management of disease [15].

\subsection{Treatment of the Underlying Disease}

Faced with a suspicion of APL (prior to final diagnosis) and a manifest coagulopathy, there are a evidence level $\mathrm{Ib}$ and a degree of recommendation A to start immediately treatment with All-trans Retinoic Acid (ATRA) [16]. Such choice will then be continued or suspended when the suspicious will it be, respectively, confirmed or refuted [17]. 


\subsection{Blood Transfusion}

Infuse concentrated red blood cells in order to maintain values of hemoglobin of between 6 and $10 \mathrm{~g} / \mathrm{dL}$. As for the infusion of platelets, in the first place exclude with certainty a TTP or heparin-induced thrombocytopenia (HIT). At this point assess the risk of bleeding: in numerical terms the bleeding risk is taken for platelet count $<$ $20,000-30,000 \mathrm{~mL}$. It is good, then, implement the platelet support with a target up to 50,000 $\mathrm{mL}$ and no further.

The fresh frozen plasma (FFP) is perhaps the most widely therapeutic remedy it employed, but it is also the most controversial and is not recommended since is implicated in the pathogenesis of transfusion related acute lung injury (TRAIL).

The cryoprecipitate should be the best formulation support with reasonable target values of fibrinogen comprised between 50 and $100 \mathrm{mg} / \mathrm{dL}$. Cryoprecipitate is rich in fibrinogen, factor VIII and VWF. It's estimated that 10 bags of cryoprecipitate can raise levels of fibrinogen of $60-100 \mathrm{mg} / \mathrm{dL}$.

It's been finally reported the usefulness of employment of the recombinant factor VII to correct situation of severe bleeding that tend to compromise quickly the patient's life, such as bleeding brain [18].

\subsection{Heparin}

When prevail thrombotic events, heparin is indicated and the low molecular weight heparin (LMWH) is preferable. Controversial is the use of antithrombin III (AT III).

\subsection{Natural Protease Inhibitor}

The recombinant human activated protein $\mathrm{C}$ (drotrecogin-alpha) is effective in case of severe sepsis. The guidelines will suggest the use in septic shock with APACHE score $>25$ [19]. The clinical use of recombinant thrombomodulin (rhTM) is not accepted by all authors. Treatment with AT III and rhTM is recommended in DIC with organ failure [20].

\subsection{Antifibrinolytic Treatment}

Their only indication concerns patients with major bleeding, particularly those with APL. In this condition the association ATRA/tranexamic acid may cause serious thrombosis [21].

\section{Hypercalcemia}

Hypercalcemia is present in $44.1 \%$ of cancer patients. With reference to hematological malignancies, multiple myeloma is most frequently responsible, followed by acute leukemias and aggressive lymphomas [22].

In $80 \%$ of cases, hypercalcemia is caused by PTH-related peptide secretion. This conduces to bone reabsorption and elevated serum calcium due to the action of osteoclasts activated by the release of RANKL by osteoblasts. Secondly, this peptide causes increased calcium reabsorption in the renal tubule [23]. In $20 \%$ of cases it is the result of osteolytic metastases, mechanism especially involved in multiple myeloma. In a minority of cases, as in lymphomas, it is due to ectopic production of 1-alpha hydroxylase and formation of 1.25 dihydroxycholecalciferol.

The main clinical manifestations are summarized in Table 2. These clinical manifestations are related both to the calcium concentration in the serum is the velocity of occurrence of hypercalcemia.

Table 2. Clinical manifestations of hypercalcemia.

\begin{tabular}{|c|c|}
\hline Organ system & Clinical features \\
\hline Neurologic & Fatigue, altered mental status including coma, posterior reversible leukoencephalopathy \\
\hline Gastrointestinal & Nausea, vomiting, constipation, peptic ulcer disease, and pancreatitis \\
\hline Cardiovascular & $\begin{array}{c}\text { Short QT interval on } \mathrm{ECG}^{\circ}, \mathrm{ST}_{\text {segment abnormalities including ST segment myocardial infarction mimic, }} \\
\text { malignant ventricular arrhythmias, and hypertension }\end{array}$ \\
\hline Renal & Nephrogenic diabetes insipidus, acute kidney injury \\
\hline
\end{tabular}

${ }^{\circ} \mathrm{ECG}=$ Electrocardiogram. 
Hypercalcemia's treatment can be obtained by arresting the pathological bone reabsorption, increasing the calcium renal excretion or reducing the calcium intestinal absorption.

In the case of asymtomatic or mild hypercalcemia (serum calcium $<12 \mathrm{mg} / \mathrm{dL}$ or $3 \mathrm{mmol} / \mathrm{L}$ ) a supportive treatment including proper hydration with saline, furosemide, and diet low in calcium may be enough waiting to treat the underlyng disease.

The treatment of moderate hypercalcemia (serum calcium $12-14 \mathrm{mg} / \mathrm{dL}$ or $3-3.5 \mathrm{mmol} / \mathrm{L}$ ) to severe (serum calcium $>14 \mathrm{mg} / \mathrm{dL}$ or $>3.5 \mathrm{mmol} / \mathrm{L}$ ) must be more quick and aggressive.

The first treatment strategy includes the volume expansion realized with the infusion of saline solution bolus (1 - 2 L) followed by maintenance at the rate of $100-150 \mathrm{ml} / \mathrm{h}$ in order to obtain a urinary excretion of $100 \mathrm{ml} / \mathrm{h}$. If this is not obtained, the addition of furosemide at a dose of $40 \mathrm{mg}$ intravenously can be realized. The use of calcitonin intramuscularly or subcutaneously to $4-8 \mathrm{IU} / \mathrm{kg}$ dose is the second treatment option. Bisphosphonates act at various levels on mechanisms responsible of bone reabsorption obtaining a rapid reduction in serum calcium. The most used are: zoledronic acid (4 mg IV over 15 - $30 \mathrm{~min}$ ) or pamidronate (60 - $90 \mathrm{mg}$ IV over 2 - $24 \mathrm{~h}$ ). The main limiting factor is the nephrotoxicity. They can be used safely in patients undergoing hemodialysis.

It can reduce bone reabsorption through the inhibition of the osteoclasts, thanks to denosumab, a monoclonal antibody that acts by forming immune complexes with RANK ligand (RANKL), protein that acts as the primary signal in the promotion of bone removal by binding to the receptor RANK. It effective in treating refractory hypercalcemia and it has the advantage of less nephrotoxicity. Denosumab is normally administered at a dose of $120 \mathrm{mg}$ subcutaneous possibly every week. Some authors suggest the use of a lower dose in patients with impaired renal function. The therapeutic effect of this drug is slower than bisphosphonates.

The most feared complication of treatment with denosumab or bisphosphonates is osteonecrosis of the jaw, although this is reported more when these drugs are used on an ongoing basis.

Other drugs less used in the treatment of hypercalcemia are cinacalcet and gallium nitrate. The first was developed to treat hypercalcemia associated with chronic renal failure and acts on the calcium sensing receptors of parathyroid cells, bone and kidney. The second inhibits osteoclast activity and increases renal calcium excretion [24].

In patients refractory to all treatments, the use of hemodialysis is recommended.

\section{Hyperleucocytosis}

Hyperleucocytosis is arbitrarily defined as a leucocyte count greater than $100 \times 10^{9} / \mathrm{L}$, and typically appears in some haematological diseases.

The morbidity and the mortality are due to the leucostasis. The number of leucocytes necessary for leucostasis changes with each pathology, owing to, among other factors, morphological, molecular and plasticity characteristics of the blast cells, as well as the capacity of endothelial cell to release cytokines.

In acute myeloid leukaemia, leucostasis appears at leucocyte counts above $100 \times 10^{9} / \mathrm{L}$, and severe symptoms appear above $400 \times 10^{9} / \mathrm{L}$ in acute lymphoblastic leukaemia.

By contrast, in chronic lymphocytic leukemia the leucostasis occurs for values between $500 \times 10^{9}$ and $1000 \times$

$10^{9}$ lymphoid elements/L. The most frequently involved organs are the brain (risk of bleeding) and the lung (risk of respiratory distress), more rarely the gastrointestinal tract. At the base of the treatment there are:

- adequate hydration and premedication with allopurinol or rasburicase;

- hydroxyurea (50 - $100 \mathrm{mg} / \mathrm{kg} /$ day) which results a rapid cytoreduction in myeloid leukemia [25];

- specific chemotherapy;

- leukocytapheresis: in cases of hyperleucocytosis secondary to AML with signs of leucostasis; the use in ALL is controversial [26].

\section{Hyperviscosity}

The most frequent conditions in which it has a blood hyperviscosity (HVS) are leukemia with hyperleucocytosis; polycitemia vera; Waldenstrom's macroglobulinemia (IgM); and multiple myeloma (IgG3, IgA and light chains).

Clinically HVS can arise from a variety of symptoms, but particularly skin and mucosal bleeding, in addition to various neurological disorders up to coma.

Thromboembolic complications are more frequent in PV. In leukemia the best treatment is represented by 
chemotherapy; if indicated, the leukapheresis can help to overcome emergencies. Phlebotomy and aspirin, in some patients cytoreduction with hydroxyurea, are the cornerstones of treatment of PV.

Because IgM has prevailing intravascular distribution (80\%), plasmapheresis is very effective in Waldenstrom's macroglobulinemia, especially at the beginning of the treatment with rituximab, to avoid the "flare" consequent to use of this drug, in particular higher viscosity of $3.5 \mathrm{cp}$ or IgM concentrations of $5 \mathrm{~g} / \mathrm{dl}$ [27].

\section{Acute Tumor Lysis Syndrome}

The acute tumor lysis syndrome (ATLS) is an oncologic emergency that may arise at the beginning of cytotoxic chemotherapy, especially in the case of tumors by high kinetic, high dimension or with high metastatic load. It is supported by the massive release of large quantities of nucleic acids, phosphate and potassium by the tumor cells destroyed.

In conditions of marked hyperleucocytosis also the only steroid therapy and/or radiant can unleash it, so like a marked fever. In the broad sense of the term chemotherapy they should also include the purine analogs and monoclonal drugs such as rituximab (although the latter so uncommon).

Cairo-Bishop definitions [28]:

1) Laboratory abnormalities (at least two simultaneous changes from $\underline{3}$ days before to $\underline{\underline{Z}}$ days after the debut of therapy):

- more than $25 \%$ increase: uric acid, potassium and phosphorus and/or

- greater than $25 \%$ reduction in serum calcium.

2) Clinical issues:

- kidney failure

- seizure

- cardiotoxicity

The critical stratification of patients according to risk classes is summarized in Table 3.

Table 3. Different classes of risk in acute tumor lysis syndrome.

\begin{tabular}{ccc}
\hline Very high risk & High risk & Intermediate risk \\
\hline lymphomas and leukemias with high $\mathrm{LDH}^{*}$ and renal failure & acute leukemias with hyperleucocytosis & others \\
& lymphomas with bulky \\
& Burkitt's lymphoma \\
\hline
\end{tabular}

${ }^{*} \mathrm{LDH}=$ lactate dehydrogenase.

The management of ATLS is the prevention!

Treatment of patients at high risk [29]:

* Hydration

Infusion of liquids in continuous in order to ensure an adequate renal flow $(100-200 \mathrm{ml} / \mathrm{h})$, from $24-48$ hours before start of therapy and continue until needed, watching daily diuresis, body weight, renal function and serum electrolytes.

* Diuretics

If signs of circulatory overload and/or decrease urine output from the input, we proceed to diuretic therapy (furosemide).

\section{* Hyperuricemia}

The day before the chemotherapy is administered allopurinol $600 \mathrm{mg} /$ day which may then be reduced to 300 $\mathrm{mg} /$ day. The main caveat in the use of allopurinol is the possible triggering effect a Stevens-Johnson syndrome. In severe hyperuricemia conditions or when accompanies kidney failure must be used rasburicase $0.2 \mathrm{mg} / \mathrm{kg}$ infused intravenously over 30 minutes.

\section{* Hyperkalemia}

It is the most dangerous complication, for the risk of induction of cardiac arrhythmias and sudden death. If appears, it should be treated immediately with the infusion of $50 \mathrm{ml}$ glucose at $50 \%$ with $8 \mathrm{U}$ of insulin, calcium gluconate and beta-2 agonists. If you do not respond to treatment, it will be initiated hemodialysis. 


\section{* Hyperphosphatemia}

If severe and symptomatic should be treated with hydration, to promote renal excretion of phosphorus.

\section{* Hypocalcemia}

Responsible of potentially serious arrhythmias and neuromuscular excitability, its treatment requires the use of calcium gluconate intravenously.

For patients at very high risk, as well as written to the high risks, it should be added the need for prior consultation with the nephrologist and for the availability immediate for emergency dialysis. For patients at intermediate risk is sufficient premedication of 24 hours with allopurinol and bland hydration.

\section{Spinal Cord Compression}

Spinal cord compression from metastatic origin is an oncologic emergency. In the absence of therapeutic treatment, this condition can result, in the short to medium term, a devastating neurological complication. In hematology, multiple myeloma and lymphoma can determine this condition in $5 \%-10 \%$, usually following to the progression of neoplastic lesion paraspinal that invades the epidural space.

The ease with which metastases develop in the spine can be explained by the fact that it is a bony region highly vascularized and rich in growth factors. The venue affected will be thoracic in $70 \%$, lumbosacral in $20 \%$ et cervical in $10 \%$ of cases.

The clinical suspect will be born by the presence of pain that can preceded by weeks or months the sensory and motor deficits, and sphincteric alterations.

The magnetic resonance imaging (MRI) is the method of choice for detecting spinal compression (sensitivity 93\%, specificity 97\%, efficiency 95\%). Prompt recognition of this dramatic complication will be critical to save the spinal cord.

Treatment includes:

- Corticosteroids: it does not exist at this time enough data to guide our choices, nor the optimum dosage nor the ideal duration of administration are known; in practice, many clinicians administer a starting dose of 10 $\mathrm{mg}$ of dexamethasone, followed by $4 \mathrm{mg}$ every six hours for a few days and then decreasing doses.

- Radiotherapy: currently, particularly in North America, a total dose of 30 Gy in 10 fractions is one of the most commonly used schemes; in Europe, shorter schemes (a dose of 8 Gy or 20 Gy in 5 fractions) are also frequently used.

- Decompressive surgery: the role of decompressive surgery remains unclear. Surgery followed by radiotherapy had allowed $84 \%$ of patients maintain the ability to walk versus $57 \%$ in the single arm radiotherapy.

Of course, chemotherapy and bisphosphonates will be used as soon as possible.

\section{Superior Vena Cava Syndrome}

The superior vena cava syndrome (SVCS) complicates the course of mediastinal tumors and is an emergency if it is associated with laryngeal or cerebral edema.

In the haematological setting the SVCS is most common in high-grade NHL, followed, in order of frequency, by Hodgkin's disease and T-cells lymphoblastic lymphoma/leukemia.

According Kishi, we can group the symptoms and signs in:

1) Respiratory: stridor, dysphagia, dyspnea, orthopnea, laryngeal edema, chest pain, coughing or pleurisy.

2) Neurological: malaise, mental disorders, visual disorders, headache, vertigo, awareness disorders or coma.

3) Facial: swelling, lip edema, nasal obstruction or epistaxis.

4) Vessel dilatation: neck, face or arms.

The differential diagnosis includes lung cancer, thymoma, sarcoma, extragonadal germ cell tumors, mediastinal metastatic tumors, aortic aneurysm and tuberculosis.

The diagnostic process is long and includes many exams, such as various cancer markers, contrast-enhanced spiral or multi-slice CT imaging and contrast-enhanced magnetic resonance (MR) venography.

From a therapeutic point of view in the past it is used radiotherapy even in the absence of pathological diagnosis. Today urgent radiotherapy is limited to less than $5 \%$ of cases, for such patients whose clinical picture is characterized by severe respiratory deficiency and is not allowed any therapeutic delay. For these critical patients there is an indication to urgent venography, superior vena cava stenting placement, and thrombolytic therapy if indicated [30]. 
Medical treatment consists of corticosteroids, chemotherapy and oxygen.

\section{Conclusion}

In hematology, there are various clinical conditions both benign and malignant needing an prompt diagnostic assessment, a rapid prognostic stratification in order to immediate therapeutic correction. The knowledge of most hematological emergencies is crucial in daily practice.

\section{Conflict of Interest}

The author has nothing to disclose and indicates no potential conflict of interest.

\section{References}

[1] Garratty, G. (2010) Drug-Induced Immune Hemolytic Anemia. Clinical Advances in Hematology and Oncology, 8, 98101.

[2] Sadler, J.E. (2015) What's New in the Diagnosis and Pathophysiology of Thrombotic Thrombocytopenic Purpura. Hematology: American Society of Hematology Education Program, 2015, 631-636.

[3] Khatun, H. and Morshed, M. (2015) Thrombotic Thrombocytopenic Purpura: Issues in Diagnosis and Treatment. Mymensingh Medical Journal, 24, 761-764.

[4] Selvi Sabater, P., Espuny Miró, A., Rizo Cerdá, A.M., et al. (2016) Cyclosporin Induced Thrombotic Thrombocytopenic Purpura. Report of Three Cases. Med Clin (Barc), 146, 231-232.

[5] Garcia, G. and Atallah, J.P. (2016) Antineoplastic Agents and Thrombotic Microangiopathy. Journal of Oncology Pharmacy Practice. http://dx.doi.org/10.1177/1078155216628324

[6] Rock, G.A., Shumak, K.H., Buskard, N.A., et al. (1991) Comparison of Plasma Exchange with Plasma Infusion in the Treatment of Thrombotic Thrombocytopenic Purpura. Canadian Apheresis Study Group. The New England Journal of Medicine, 325, 393-397. http://dx.doi.org/10.1056/nejm199108083250604

[7] Omri, H.E., Taha, R.Y., Gamil, A., et al. (2015) Efficacy and Safety of Rituximab for Refractory and Relapsing Thrombotic Thrombocytopenic Purpura: A Cohort of 10 Cases. Clinical Medicine Insights: Blood Disorders, 8, 1-7.

[8] Peyvandi, F., Scully, M., Kremer Hoving, J.A., et al., TITAN Investigators (2016) Caplacizumab for Acquired Thrombotic Thrombocytopenic Purpura. The New England Journal of Medicine, 374, 511-522. http://dx.doi.org/10.1056/NEJMoa1505533

[9] Sayani, F.A. and Abrams, C.S. (2015) How I Treat Refractory Thrombotic Thrombocytopenic Purpura. Blood, 125, 3860-3867. http://dx.doi.org/10.1182/blood-2014-11-551580

[10] Wang, C. (2004) Sickle Cell Anemia and Other Sickling Sindromes. In: Wintrobe's Clinical Hematology, 11th Edition, Lippincott Williams and Wilkins, Philadelphia, 1262-1311.

[11] Novelli, E.M. and Gladwin, M.T. (2016) Crises in Sickle Cell Disease. Chest, 149, 1082-1093. http://dx.doi.org/10.1016/j.chest.2015.12.016

[12] Wada, H. (2004) Disseminated Intravascular Coagulation. Clinica Chimica Acta, 344, 13-21. http://dx.doi.org/10.1016/j.cccn.2004.02.015

[13] Taylor, F.B.Jr., Toh, C.H., Hoots, W.K., et al. (2001) Scientific Subcommittee on Disseminated Intravascular Coagulation (DIC) of the International Society on Thrombosis and Haemostasis (ISTH) towards Definition, Clinical and Laboratory Criteria, and a Scoring System for Disseminated Intravascular Coagulation. Thrombosis and Haemostasis, 86, 1327-1330.

[14] Wada, H., Thachil, J., Di Nisio, M., et al. (2013) The Scientific Standardization Committee on DIC of the International Society on Thrombosis Haemostasis. Guidance for Diagnosis and Treatment of DIC from Harmonization of the Recommendations from Three Guidelines. Journal of Thrombosis and Haemostasis. http://dx.doi.org/10.1111/jth.12155

[15] Levi, M., Toh, C.H., Thachil, J., et al. (2009) Guidelines for the Diagnosis and Management of Disseminated Intravascular Coagulation. British Committee for Standards in Haematology. British Journal of Haematology, 145, $24-33$. http://dx.doi.org/10.1111/j.1365-2141.2009.07600.x

[16] Sanz, M.A., Grimwade, D., Tallman, M.S., et al. (2009) Management of Acute Promyelocytic Leukemia: Recommendations from an Expert Panel on Behalf of the European Leukemia Net. Blood, 113, 1875-1891. http://dx.doi.org/10.1182/blood-2008-04-150250

[17] De la Serna, J., Montesinos, P., Vellenga, E., et al. (2008) Causes and Prognostic Factors of Remission Induction Failure in Patients with Acute Promyelocytic Leukemia Treated with All-Trans Retinoic Acid and Idarubicin. Blood, 111, 
3395-3402. http://dx.doi.org/10.1182/blood-2007-07-100669

[18] Zver, S., Andoljsek, D. and Cernelc, P. (2004) Effective Treatment of Life Threatening Bleeding with Recombinant Activated Factor VII in a Patient with Acute Promyelocyticleukaemia. European Journal of Haematology, 72, 455456. http://dx.doi.org/10.1111/j.1600-0609.2004.00237.x

[19] Ranieri, V.M., Thompson, B.T., Barie, P.S., et al. (2012) PROWESS-SHOCK Study Group. Drotrecogin-alfa (Activated) in Adults with Septic Shock. New England Journal of Medicine, 366, 2055-2064. http://dx.doi.org/10.1056/NEJMoa1202290

[20] Yamakawa, K., Fujimi, S., Mohri, T., et al. (2011) Treatment Effects of Recombinant Human Soluble Thrombomodulin in Patients with Severe Sepsis: A Historical Control Study. Critical Care, 15, R123. http://dx.doi.org/10.1186/cc10228

[21] Brown, J.E., Olujohungbe, A., Chang, J., et al. (2000) All-Trans Retinoic Acid (ATRA) and Tranexamic Acid: A Potentially Fatal Combination in Acute Promyelocyticleukaemia. British Journal of Haematology, 110, 1010-1012. http://dx.doi.org/10.1046/j.1365-2141.2000.02270-8.x

[22] Stewart, A.F. (2005) Clinical Practice. Hypercalcemia Associated with Cancer. New England Journal of Medicine, 352, 373-379. http://dx.doi.org/10.1056/NEJMcp042806

[23] Wysolmerski, J.J. (2012) Parathyroid Hormone-Related Protein: An Update. Journal of Clinical Endocrinology and Metabolism, 97, 2947-2956. http://dx.doi.org/10.1210/jc.2012-2142

[24] Sternlicht, H. and Glezerman, I.G. (2015) Hypercalcemia of Malignancy and New Treatment Options. Therapeutics and Clinical Risk Management, 11, 1779-1788.

[25] Grund, F.M., Armitage, J.O. and Burns, P. (1997) Hydroxyurea in the Prevention of the Effects of Leukostasis in Acute Leukemia. Archives of Internal Medicine, 137, 1246-1267. http://dx.doi.org/10.1001/archinte.1977.03630210112032

[26] Parra-Salinas, I.M., González Rodriguez, V.P. and García-Erce, J.A. (2015) Therapeutic Leukapheresis: 9-Year Experience in a University Hospital. Blood Transfusion, 13, 46-52.

[27] Stone, M.J. and Bogen, S.A. (2012) Evidence-Based Focused Review of Management of Hyperviscosity Syndrome. Blood, 119, 2205-2208. http://dx.doi.org/10.1182/blood-2011-04-347690

[28] Wilson, F.P. and Berns, J.S. (2014) Tumor Lysis Syndrome: New Challenges and Recent Advances. Advances in Chronic Kidney Disease, 21, 18-26. http://dx.doi.org/10.1053/j.ackd.2013.07.001

[29] Mirrakhimov, A.E., Voore, P., Khan, M. and Ali, A.M. (2015) Tumor Lysis Syndrome: A Clinical Review. World Journal of Critical Care Medicine, 4, 130-138. http://dx.doi.org/10.5492/wjccm.v4.i2.130

[30] Straka, C., Ying, J., Kong, F.M., et al. (2016) Review of Evolving Etiologies, Implications and Treatment Strategies for the Superior Vena Cava Syndrome. Springer Plus, 5, 229. http://dx.doi.org/10.1186/s40064-016-1900-7

\section{Submit or recommend next manuscript to SCIRP and we will provide best service for you:}

Accepting pre-submission inquiries through Email, Facebook, LinkedIn, Twitter, etc.

A wide selection of journals (inclusive of 9 subjects, more than 200 journals)

Providing 24-hour high-quality service

User-friendly online submission system

Fair and swift peer-review system

Efficient typesetting and proofreading procedure

Display of the result of downloads and visits, as well as the number of cited articles

Maximum dissemination of your research work

Submit your manuscript at: http://papersubmission.scirp.org/ 